\title{
Urinary Calprotectin as a Marker to Distinguish Functional and Structural Acute Kidney Injury in Pediatric Population
}

\author{
Mitra Basiratnia, ${ }^{1,}$ Masrur Kosimov, ${ }^{1}$ Pouya Farhadi, ${ }^{2}$ Arsalan Azimi, ${ }^{2}$ and Nakysa Hooman ${ }^{3}$ \\ ${ }^{1}$ Shiraz Nephrology Urology Research Center Shiraz University of Medical Sciences, Shiraz, Iran \\ ${ }^{2}$ Student Research Committee, School of Medicine, Shiraz University of Medical Sciences, Shiraz, Iran \\ ${ }^{3}$ Ali-Asghar Children Hospital, Iran University of Medical Sciences, Tehran, Iran \\ "Corresponding author: Mitra Basiratnia, Pediatric Department, Namazi Hospital, Shiraz, Iran. Tel: +98-9177875341, E-mail: m_basirtnia@yahoo.com
}

Received 2016 November 18; Revised 2017 April 09; Accepted 2017 May 07.

\begin{abstract}
Background: Acute kidney injury (AKI) is a serious, common and occasionally under-recognized condition. To date, the clinical and some laboratory parameters are routinely applied to distinguish between functional and structural AKI which can be challenging in certain occasions. In the present paper, we investigate the accuracy of urinary calprotectin as a diagnostic biomarker in this dubious situation.

Methods: This is a cross-sectional study among 75 children with AKI defined by Acute Kidney Injury Network (AKIN) and 20 healthy children as controls which was carried out for about six months (September 2014 to March 2015). Random urinary calprotectin concentration was assessed by ELISA in both groups within 48 hours after diagnosis. Patients with obstructive uropathy, malnutrition, renal transplantation, chronic renal failure, urinary tract infection, and malignancy were excluded. Receiver-operating characteristic (ROC) curves were drawn to determine the accuracy of urinary calprotectin to detect children with structural AKI. P value less than 0.05 was considered significant.

Results: Median urinary calprotectin was $1240 \mathrm{ng} / \mathrm{mL}$ in structural AKI, 28.5 in functional, and 33 in controls. Receiver operating curve analysis revealed high levels of accuracy for measuring calprotectin in predicting structural AKI. A cutoff level of $230 \mathrm{ng} / \mathrm{mL}$ for urinary calprotectin showed high sensitivity and specificity. The urine calprotectin/creatinine ratio indicated the same accuracy as urinary calprotectin in diagnosing structural AKI. The ROC curve function was better for urine calprotectin and its ratio in comparison to fractional excretion of sodium (FENa).

Conclusions: Calprotectin is a biomarker that can rapidly and easily recognize structural from functional AKI with high sensitivity and specificity in comparison to traditional most accurate diagnostic test; FENa.
\end{abstract}

Keywords: Acute Kidney Injury, Structural, Calprotectin, Functional, Children

\section{Background}

Acute kidney injury (AKI) is a difficult prevalent disease that accounts for approximately $50 \%$ of consultations with nephrologist (1). The incidence is highly dependent on the setting, hospital vs. community acquired AKI. The severity and duration of AKI predicts its outcome (2). Although it is more prevalent in PICU; the new concern is medication associated AKI in non-ill children (3). After high nephrotoxic drug exposure residual damage and chronic kidney disease (CKD) have been demonstrated in two thirds of AKI patients after six months follow up (4).

AKI is also associated with longer hospitalization time, higher rate of mortality, and increased risk of chronic kidney disease in adulthood. As such, early diagnosis and treatment can potentially improve the outcome $(5,6)$. Unfortunately early distinction between functional and structural AKI is difficult on the basis of clinical findings and conventional laboratory parameters such as urine output, urine osmolality, fractional excretion of sodium, BUN to Cr ratio, urine sediment, etc. (7). The aim of a number of studies was to find more sensitive biomarkers to recognize AKI in the earliest stage. Biomarkers rise in different timepoints of the kidney injury and each biomarker individually will not perform well in various types of AKI (8-10). Although a panel of all biomarkers perform the best to detect AKI in early or different stages; the present study was designed by emphasis on random measurement of a single biomarker. All new biomarkers have some limitations such as the influence of infection, durability of biomarker, and low precision in distinction of acute from chronic kidney disease (11).

Among all biomarkers, Neutrophil GelatinaseAssociated Lipocalin (NGAL) has most widely been studied. NGAL has high specificity to distinguish structural AKI from CKD, normal population and hypovolemic states, better prediction of excessive morbidity (12), the most powerful marker indicating the severity and duration of 
AKI (13), and an accurate marker for early detection of AKI in high risk cases such as sepsis patients $(14,15)$.

Calprotectin is a mediator protein of the innate immune system formed by complex of S100A8 and S100A9, two immune-regulatory calcium-binding proteins, found mainly in neutrophils and to a lesser extent in monocytes and reactive macrophages (16) and it is shown to protect against oxidative stress during inflammation (17). Additionally, renal collecting duct epithelial cells produce calprotectin and this marker is detectable in urine early after renal injury (18).

There is an increase in the amount of calprotectin in instances such as urinary tract obstruction, infection, rheumatoid arthritis, inflammatory bowel diseases, urethral carcinoma, bladder cancer, and probably in chronic kidney disorders $(11,19)$. There are few studies conducted in cisplatin induced ischemic kidney in rat and adult that show calprotectin could differentiate between pre-renal and ischemic acute kidney injury (20-24).

It has been shown that urinary calprotectin was superior to creatinine $(\mathrm{Cr})$ for prediction of early renal ischemic injury and future renal function in patients with renal transplantation $(25,26)$. Moreover, animal study revealed that calprotecin has an essential role in development and severity of glomerulonephritis (27). Recently, Westhoff et al. studied the urinary biomarkers to differentiate between intrinsic and pre-renal AKI in children and found out that urinary calprotectin is a better detector (28).

Regarding the pitfalls for historic laboratory gold standard, FENa (in post ischemic ATN, ATN superimposed upon a chronic pre-renal disease, AKI due to radio contrast or pigments, acute glomerulonephritis, interstitial nephritis, and concurrent diuretic usage) and the paucity of pediatric studies, we conducted this study to evaluate the accuracy of calprotectin in differentiation between functional and structural AKI in pediatric population.

\section{Methods}

This is a prospective cross-sectional study conducted for a period of six months (September 2014 and March 2015) in two training hospitals (Namazi and Shahid Dastgheib) affiliated to Shiraz University of Medical Sciences. Seventy-five patients, aged from three months to 18 years, with diagnosis of acute kidney injury recruited from PICU, ward, and emergency room were enrolled in the study. Renal biopsy of all children with glomerular disease revealed accompanying tubulointerstitial inflammation. Twenty healthy children with no history of renal diseases, hypertension, or diabetes mellitus who had come to clinic for regular check-up were also included as control.
The study was approved by Ethics committee of Shiraz University of Medical Sciences (ID number 93-01-01-7154 dated 22.10.2015). Following explanation of the whole protocol in details, written consent was taken from parents before entering their children into the study.

AKI was defined by abrupt increment of serum $\mathrm{Cr}$ at least $0.3 \mathrm{mg} / \mathrm{dL}$ from the baseline serum $\mathrm{Cr}$ within 48 hours of assessment (29).

Patients were categorized into two groups of functional (pre-renal) and structural (intrinsic) AKI. Structural AKI included ischemic injury, sepsis, glomerulonephritis, and hemolytic uremic syndrom. Decline in serum $\mathrm{Cr}$ to the baseline after fluid repletion in less than 48 hours was attributed to functional (pre-renal) injury.

The exclusion criteria were obstructive uropathy, urinary tract infection, malnutrition, renal transplantation, chronic kidney diseases, and malignancy.

Serum $\mathrm{Cr}$ was measured by Jaffe method. eGFR was calculated by Schwartz formula $(($ Height $(\mathrm{cm}) \times 0.55) /$ Plasma $\mathrm{cr}(\mathrm{mg} / \mathrm{dL}))(30)$. Serum $\mathrm{Cr}$ level was measured at the time of zero of entrance and then daily, afterwards. In our laboratory it takes three hours from the blood draw to receive the results of serum $\mathrm{Cr}$.

Having had the diagnosis of AKI; urine sample $(10 \mathrm{~mL})$ would be collected simultaneously with blood sample on the second day of admission. Then, the samples were frozen $\left(-20^{\circ} \mathrm{C}\right.$, no centrifugation) for assessment of calprotectin level. Urinary levels of calprotectin were quantified using an enzyme-linked immunosorbent assay (ELISA) kit (PhiCal ${ }^{\circledR}$ Calprotectin, catalogue number K 6928; Immundiagnostik AG, Bensheim, Germany) according to the manufacturer's protocol. The coefficient of variation for duplicate measurements using this ELISA kit was $<6 \%$. To take the current concentration status of the urine into account, urinary $\mathrm{Cr}$ was assessed and then calprotectin/Cr ratio was calculated. Urine and serum sodium were measured by Flame photometry with Hycell PHF-104. Fractional excretion of sodium was calculated by using the formula $($ UNaxPcr $/$ PNaxUcr $) \times 100$. The level below $1 \%$ was considered as pre-renal and above that as intrinsic or renal AKI.

\subsection{Statistical Analyses}

Data were presented as mean \pm standard deviation (SD) or median (range) with $95 \%$ confidence intervals. The comparison of variables was done by using $\varkappa^{2}$ or Fisher's exact test for proportions and unpaired t-tests for means. A value of $\mathrm{P}<0.05$ was considered as statistically significant. Receiver-operating characteristic (ROC) curves were constructed to determine the accuracy of urinary calprotectin and calprotectin/Cr ratio to detect children with in- 
trinsic AKI. Sensitivity, specificity, and positive and negative predictive values were estimated.

\section{Results}

From among 75 children with clinical diagnosis of AKI, 45 were classified as structural and 30 as functional AKI. In addition, 20 healthy children were enrolled in the control group. Table 1 depicts the demographics data, etiology, renal parameters and calprotectin measurements in the children with AKI and controls. Mean urinary calprotectin was 36 times higher in structural than in functional AKI, and 44 times higher than those in healthy children $(\mathrm{P}<0.001)$. Additionally, there was no statistically significant difference between functional AKI and healthy subjects regarding calprotectin/Cr ratios $(\mathrm{P}=0.21)$. By considering urine calprotectin to $\mathrm{Cr}$ ratio as the distinguishing biomarker, it was revealed that the ratio in structural AKI was about 140 times higher than that in functional AKI $(\mathrm{P}<0.001)$.

We assessed the level of calprotectin, FENa and urine/plasma $\mathrm{Cr}$ in different severity of AKI. As shown in Figure 1, median urinary calprotectin was rising by intensification of the severity of AKI. $(\mathrm{P}<0.001)$. This was also true for FENa.

Receiver-operating characteristic (ROC) curves were constructed to determine accuracy of urinary calprotectin and calprotectin/Cr ratio to detect children with structural AKI (Figure 2) compared to FENa. The ROC curve function was better for urine calprotectin and its ratio to $\mathrm{Cr}$. The optimal cut off value of urinary calprotectin for differentiation of functional and structural AKI was $230 \mathrm{ng} / \mathrm{mL}$ (sensitivity: 95.6\% and specificity: 100\% positive predictive value (PPV): 100\%, negative predictive value (NPV): 93.8\%). Besides, the cut off value of urine calprotectin/Cr ratio achieved was $383 \mathrm{ng} / \mathrm{mL}$ with sensitivity and specificity of 96.7\%, PPV of 97.7\%, and NPV of 96.7\%. The ROC function was better than FENa and urine/plasma $\mathrm{Cr}$.

Even at the same level of serum $\mathrm{Cr}$ and eGFR, FENa had an overlap for the functional and structural AKI. Therefore, it was not a reliable marker to be used on its own to differentiate these two classes of AKI.

\section{Discussion}

This study shows that urinary calprotectin has a good performance by a cut-off value of $230 \mathrm{ng} / \mathrm{mL}$, in differentiation of structural and functional AKI in pediatric population with high sensitivity (95.6\%) and specificity (100\%).

The traditional definition of AKI is based on history, clinical feature, and laboratory tests. According to the results, the patients were classified into pre-renal (functional), post-renal, or structural (intrinsic) AKI. The implication of such criteria for accurate diagnosis has some limitations. These parameters might be influenced by the time of drawing blood or taking urine samples, fluid therapy, diuretic therapy and even the superimposition of AKI on an underlying renal disease. Even the validity of these parameters to put a patient in functional or structural group was not verified histologically (31).

In our study, fractional excretion criteria of $1 \%$ could classify at least $15.6 \%$ patients in structural and half of the patients in functional group correctly. By considering urine/plasma $\mathrm{Cr}$, about $27 \%$ of structural and $24 \%$ of functional AKIs were misclassified.

Basu et al. studied the combination functional (plasma Cystatin C) and tubular damage (urine NGAL) biomarkers to predict the severity and persistency of AKI defined by KDGOI in 345 children after cardiopulmonary bypass. For both entities the likelihood ratios of combination biomarkers were greater than changes of serum $\mathrm{Cr}$ from baseline (32).

The presence of a biomarker and its reliability and accuracy in this challenging situation is crucial. Nicolas et al. studied the value of single measurement of NGAL in comparison to alpha1-microglobulin, alpha1-acid glycoprotein, FENa, and serum $\mathrm{Cr}$ to recognize intrinsic AKI from prerenal, CKD and healthy in 635 adults in emergency setting. The study revealed that NGAL was superior to other biomarkers and it was also predictive of the outcome (12).

There are few studies on the role of urinary calprotectin in discrimination between functional and structural AKI. The majority were done in adult setting or experimental study in animals. Heller $F$ et al. showed high accuracy of urinary calprotectin for diagnosis of intrinsic AKI. They demonstrated that the cut-off level of $300 \mathrm{ng} / \mathrm{mL}$ has a sensitivity of $92.3 \%$ and specificity of $97.1 \%$ in predicting intrinsic AKI (21).

Seibert FS et al. demonstrated that high urinary calprotectin with cutoff value of $440 \mathrm{ng} / \mathrm{mL}$ is able to differentiate between pre-renal and intrinsic AKI (22). There is no study on children but one has recently been published by Westhoff et al. They compared urinary calprotectin, NGAL and KIM-1 in AKI children defined by pRIFLE and neonates defined by KDIGO. The study indicated calprotectin was superior to two other biomarkers to recognize structural AKI (28). In our study, we found that the level of random urinary calprotectin was correlated with the severity of AKI and its median was progressively higher in injury and failure class of AKI. Yet, in the same level of $\mathrm{Cr}$ and eGFR in functional (pre-renal) and structural (intrinsic) AKI, urine calprotectin was higher in structural group (P value $<0.05$ ). These findings in our study might be helpful to discrim- 
Table 1. Demographic and Laboratory Data in Study Groups with AKI

\begin{tabular}{|c|c|c|c|c|}
\hline Variables & Functional $(\mathbf{N}=\mathbf{3 0})$ & Structural $(N=45)$ & $\operatorname{Control}(\mathrm{N}=\mathbf{2 0})$ & PValue \\
\hline Gender, No. (\%) & & & & 0.97 \\
\hline Female & $15(50)$ & $23(51.1)$ & $10(50)$ & \\
\hline Male & $15(50)$ & $22(49.9)$ & $10(50)$ & \\
\hline Age, y; median, range & $7.5(1-17)$ & $6(1-17)$ & $10(1-17)$ & 0.61 \\
\hline BMI, $\mathbf{k g} / \mathbf{m}^{2} ;$ median, range & $18.9(5-23)$ & $18.7(12-22.5)$ & $19.6(13.3-28)$ & 0.53 \\
\hline \multicolumn{5}{|l|}{ Origin of AKI, No. (\%) } \\
\hline Dehydration by gastroenteritis & $30(100)$ & & & \\
\hline Hypotension-induced ATN & & $15(33.3)$ & & \\
\hline Sepsis-induced ATN & & $15(33.3)$ & & \\
\hline Crescentic Glomerulonephritis & & $4(8.8)$ & & \\
\hline Lupus Nephritis & & $2(4.4)$ & & \\
\hline Hemolytic Uremic Syndrome & & 5(11.1) & & \\
\hline Glomerulonephritis & & $4(8.8)$ & & \\
\hline \multicolumn{5}{|l|}{ Renal parameters; median, range } \\
\hline eGFR, $\mathrm{mL} / \mathrm{min} / 1.73 \mathrm{~m}^{2}$ & $66(38-100)$ & $30(10-60)$ & $143(110-190)$ & $<0.001$ \\
\hline Pcr, mg/dL & $1.1(0.7-1.6)$ & $1.8(1.3-5)$ & $0.5(0.3-0.88)$ & $<0.001$ \\
\hline $\mathrm{PNa}, \mathrm{mmol} / \mathrm{L}$ & $141.5(136-145)$ & $141(136-145)$ & $141(136-145)$ & 0.39 \\
\hline $\mathrm{UNa}, \mathrm{mmol} / \mathrm{L}$ & $97(6-165)$ & $80(6-163)$ & $75(30-120)$ & 0.98 \\
\hline FENa, (\%) & $1(0.06-6.5)$ & $7(0.18-53)$ & $0.7(0.2-3)$ & $<0.001$ \\
\hline U Calprotectin, ng/mL & $28.5(5-230)$ & $1240(100$ - 8180), Glomerular: $1040(150$ - 7940), ATN:1260 (100 - 8180) & $33(5-210)$ & $<0.001$ \\
\hline U Calprotectin/Ucr, (ng/mL)/(mg/dL) & $94(5-628)$ & $19352(158$ - 107000), Glomerular: 2020 (166 - 56714), ATN:6800 (158 - 107000) & $155(16-8400)$ & $<0.001$ \\
\hline
\end{tabular}

Abbreviations: BMI, Body Mass Index; eGFR, Estimated Glomerular Filtration Rate; FENa, Fractional Excretion of Sodium; Pcr, Plasma Creatinin; PNa, Plasma Sodium; UNa, Urine Sodium.
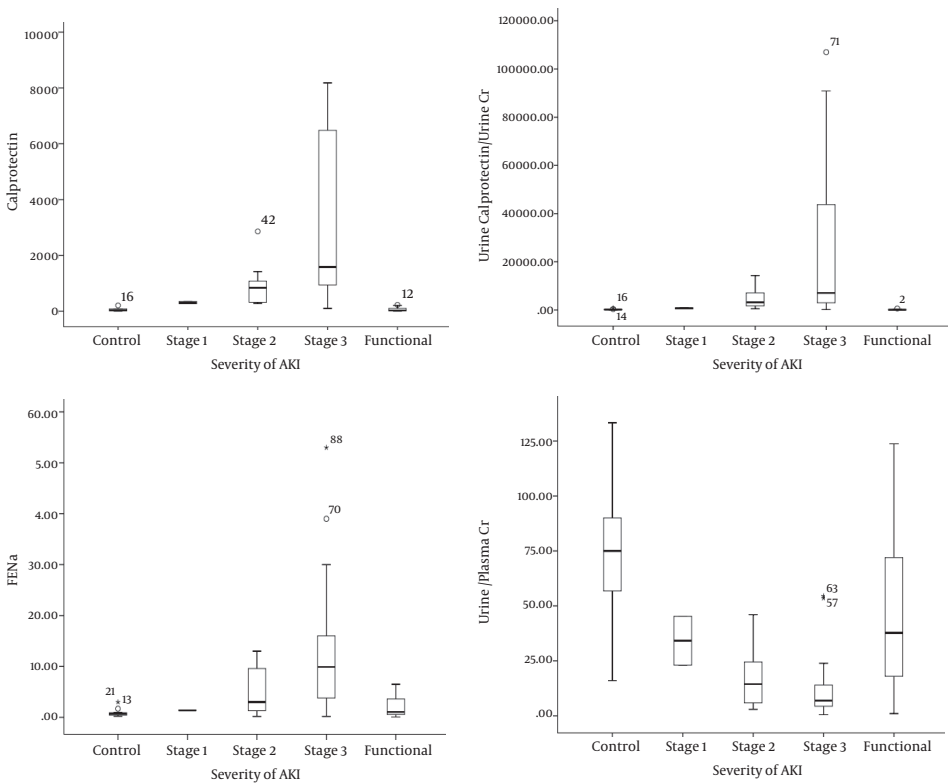

Figure 1. The Median of Traditional AKI Markers vs. Calprotectin in Various Severity of AKI $(\mathrm{P}<0.0001)$

inate these two entities especially when the traditional markers are not helpful to do the correct classification. Albeit, it might be helpful to estimate the severity of AKI.
Limitations of study were as follows: Exclusion of patients with malignancy as the levels of calprotectin have been studied in some malignancies such as breast cancer, 

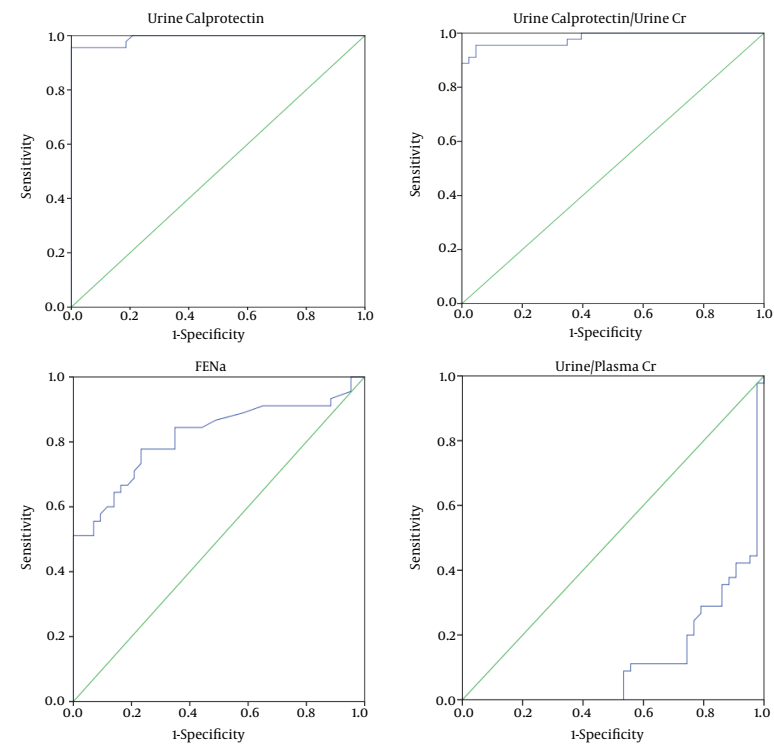

\begin{tabular}{|l|c|c|c|c|}
\hline & \multirow{2}{*}{\begin{tabular}{l}
\multirow{2}{*}{ Test Result Variable(s) } \\
Asymptoti
\end{tabular}} & \multicolumn{2}{|c|}{$\begin{array}{c}\text { Asymptotic 95\% Confidence } \\
\text { Interval }\end{array}$} \\
\cline { 4 - 5 } & Area & c Sig. & Lower Bound & Upper Bound \\
\hline Calprotectin & 0.99 & .000 & 0.98 & 1 \\
Urine & & & & 1 \\
Calprotectin/Urine cr & 0.98 & .000 & 0.96 & 0.91 \\
FENa & 0.82 & .000 & 0.72 & 0.19 \\
Urine/Plasma cr & 0.12 & .000 & 0.05 & 1 \\
\hline
\end{tabular}

Figure 2. ROC Curve Function of Traditional Marker and Urine Calprotectin to Differentiate Structural from Functional AKI or Healthy Children

urothelial and bladder carcinoma. Hence, we did not enter this group of patients due to uncertainty for the impact of underlying diseases as confounding factor for calprotectin measurement as a biomarker of AKI. Because of inadequate budget we could not measure the other new biomarkers along with urine calprotectin simultaneously. This entity may need another prospective study with combination of biomarkers; single measurement of calprotectin at the time of AKI diagnosis and lack of serial measurements, small sample sizes, lack of simultaneous measurement of NGAL, the absence of histological confirmation in the majority of study groups (except glomerulonephritis), and lack of immnuohistochemical study for calprotectin in structural AKI are other potential limitation of the present study.

\subsection{Conclusion}

Calprotectin could accurately diagnose structural from functional AKI. Its level increases in proportion with severity of AKI.

\section{References}

1. Koyner JL, Cerda J, Goldstein SL, Jaber BL, Liu KD, Shea JA, et al. The daily burden of acute kidney injury: a survey of U.S. nephrologists on World Kidney Day. Am J Kidney Dis. 2014;64(3):394-401. doi: 10.1053/j.ajkd.2014.03.018. [PubMed: 24815216].

2. Lewington AJ, Cerda J, Mehta RL. Raising awareness of acute kidney injury: a global perspective of a silent killer. Kidney Int. 2013;84(3):45767. doi: 10.1038/ki.2013.153. [PubMed: 23636171].

3. Moffett BS, Goldstein SL. Acute kidney injury and increasing nephrotoxic-medication exposure in noncritically-ill children. Clin J Am Soc Nephrol. 2011;6(4):856-63. doi: 10.2215/CJN.08110910. [PubMed: 21212419].

4. Menon S, Kirkendall ES, Nguyen H, Goldstein SL. Acute kidney injury associated with high nephrotoxic medication exposure leads to chronic kidney disease after 6 months. J Pediatr. 2014;165(3):522-7 e2. doi: 10.1016/j.jpeds.2014.04.058. [PubMed: 24928698].

5. Alkandari O, Eddington KA, Hyder A, Gauvin F, Ducruet T, Gottesman $\mathrm{R}$, et al. Acute kidney injury is an independent risk factor for pediatric intensive care unit mortality, longer length of stay and prolonged mechanical ventilation in critically ill children: a two-center retrospective cohort study. Crit Care. 2011;15(3):R146. doi: 10.1186/cc10269. [PubMed: 21663616].

6. Mehta P, Sinha A, Sami A, Hari P, Kalaivani M, Gulati A, et al. Incidence of acute kidney injury in hospitalized children. Indian Pediatr. 2012;49(7):537-42. doi: 10.1007/s13312-012-0121-6. [PubMed: 22317984].

7. Winterberg PD, Lu CY. Acute kidney injury: the beginning of the end of the dark ages. Am J Med Sci. 2012;344(4):318-25. doi: 10.1097/MAJ.ob013e318228aef8. [PubMed: 21817881]. 
8. Al-Ismaili Z, Palijan A, Zappitelli M. Biomarkers of acute kidney injury in children: discovery, evaluation, and clinical application. Pediatr Nephrol. 2011;26(1):29-40. doi: 10.1007/s00467-010-1576-0. [PubMed: 20623143].

9. Devarajan P. Biomarkers for the early detection of acute kidney injury. Curr Opin Pediatr. 2011;23(2):194-200. doi: 10.1097/MOP.ob013e328343f4dd.

10. Ostermann M, Philips BJ, Forni LG. Clinical review: Biomarkers of acute kidney injury: where are we now? Crit Care. 2012;16(5):233. doi: 10.1186/cc11380. [PubMed: 23014769].

11. Kashani K, Cheungpasitporn W, Ronco C. Biomarkers of acute kidney injury: the pathway from discovery to clinical adoption. Clin Chem Lab Med. 2017 doi: 10.1515/cclm-2016-0973. [PubMed: 28076311].

12. Nickolas TL, O'Rourke MJ, Yang J, Sise ME, Canetta PA, Barasch N, et al. Sensitivity and specificity of a single emergency department measurement of urinary neutrophil gelatinase-associated lipocalin for diagnosing acute kidney injury. Ann Intern Med. 2008;148(11):810-9. doi: 10.7326/0003-4819-148-11-200806030-00003. [PubMed: 18519927].

13. Nickolas TL, Schmidt-Ott KM, Canetta P, Forster C, Singer E, Sise M, et al. Diagnostic and prognostic stratification in the emergency department using urinary biomarkers of nephron damage: a multicenter prospective cohort study. J Am Coll Cardiol. 2012;59(3):246-55. doi: 10.1016/j.jacc.2011.10.854. [PubMed: 22240130].

14. Shapiro NI, Trzeciak S, Hollander JE, Birkhahn R, Otero R, Osborn $\mathrm{TM}$, et al. The diagnostic accuracy of plasma neutrophil gelatinaseassociated lipocalin in the prediction of acute kidney injury in emergency department patients with suspected sepsis. Ann Emerg Med. 2010;56(1):52-59 e1. doi: 10.1016/j.annemergmed.2010.02.010. [PubMed: 20363526].

15. Soto K, Papoila AL, Coelho S, Bennett M, Ma Q, Rodrigues B, et al. Plasma NGAL for the diagnosis of AKI in patients admitted from the emergency department setting. Clin J Am Soc Nephrol. 2013;8(12):205363. doi: 10.2215/CJN.12181212. [PubMed: 24009223].

16. van den Bergh FA, Kolkman JJ, Russel MG, Vlaskamp RT, Vermes I [Calprotectin: a fecal marker for diagnosis and follow-up in patients with chronic inflammatory bowel disease]. Ned Tijdschr Geneeskd. 2003;147(48):2360-5. [PubMed: 14677476]

17. Fujiu K, Manabe I, Nagai R. Renal collecting duct epithelial cells regulate inflammation in tubulointerstitial damage in mice. J Clin Invest. 2011;121(9):3425-41. doi: 10.1172/JCI57582. [PubMed: 21821915].

18. Schrezenmeier EV, Barasch J, Budde K, Westhoff T, Schmidt-Ott KM Biomarkers in acute kidney injury - pathophysiological basis and clinical performance. Acta Physiol (Oxf). 2017;219(3):554-72. doi: 10.1111/apha.12764. [PubMed: 27474473].

19. Ebbing J, Mathia S, Seibert FS, Pagonas N, Bauer F, Erber B, et al.Urinary calprotectin: a new diagnostic marker in urothelial carcinoma of the bladder. World JUrol. 2014;32(6):1485-92. doi:10.1007/s00345-013-12278. [PubMed: 24378824].

20. Kim AJ, Ro H, Kim H, Chang JH, Lee HH, Chung W, et al. Klotho and S100A8/A9 as Discriminative Markers between Pre-Renal and Intrin- sic Acute Kidney Injury. PLoS One. 2016;11(1):e0147255. doi:10.1371/journal.pone.0147255. [PubMed: 26799323].

21. Heller F, Frischmann S, Grunbaum M, Zidek W, Westhoff TH. Urinary calprotectin and the distinction between prerenal and intrinsic acute kidney injury. Clin J Am Soc Nephrol. 2011;6(10):2347-55. doi 10.2215/CJN.02490311. [PubMed: 21885792].

22. Seibert FS, Pagonas N, Arndt R, Heller F, Dragun D, Persson P, et al. Calprotectin and neutrophil gelatinase-associated lipocalin in the differentiation of pre-renal and intrinsic acute kidney injury. Acta Physiol (Oxf). 2013;207(4):700-8. doi: 10.1111/apha.12064. [PubMed: 23336369].

23. Ebbing J, Seibert FS, Pagonas N, Bauer F, Miller K, Kempkensteffen C, et al. Dynamics of Urinary Calprotectin after Renal Ischaemia. PLoS One. 2016;11(1):e0146395. doi: 10.1371/journal.pone.0146395. [PubMed: 26745147].

24. Chang $\mathrm{CH}$, Yang $\mathrm{CH}$, Yang $\mathrm{HY}$, Chen $\mathrm{TH}$, Lin $\mathrm{CY}$, Chang SW, et al. Urinary Biomarkers Improve the Diagnosis of Intrinsic Acute Kidney Injury in Coronary Care Units. Medicine (Baltimore). 2015;94(40):e1703. doi: 10.1097/MD.0000000000001703. [PubMed: 26448023].

25. Tepel M, Borst C, Bistrup C, Marcussen N, Pagonas N, Seibert FS, et al Urinary calprotectin and posttransplant renal allograft injury. PLoS One. 2014;9(11):e113006. doi: 10.1371/journal.pone.0113006. [PubMed: 25402277].

26. Szeto CC, Kwan BC, Lai KB, Lai FM, Chow KM, Wang G, et al. Urinary expression of kidney injury markers in renal transplant recipients. Clin J Am Soc Nephrol. 2010;5(12):2329-37. doi: 10.2215/CJN.01910310. [PubMed: 20671224].

27. Pepper RJ, Wang HH, Rajakaruna GK, Papakrivopoulou E, Vogl T, Pusey CD, et al. S100A8/A9 (calprotectin) is critical for development of glomerulonephritis and promotes inflammatory leukocyte-renal cell interactions. Am J Pathol. 2015;185(5):1264-74. doi: 10.1016/j.ajpath.2015.01.015. [PubMed: 25759267].

28. Westhoff JH, Fichtner A, Waldherr S, Pagonas N, Seibert FS, Babel N, et al. Urinary biomarkers for the differentiation of prerenal and intrinsic pediatric acute kidney injury. Pediatr Nephrol. 2016;31(12):2353-63. doi: 10.1007/s00467-016-3418-1. [PubMed: 27286687].

29. Mehta RL, Kellum JA, Shah SV, Molitoris BA, Ronco C, Warnock DG et al. Acute Kidney Injury Network: report of an initiative to improve outcomes in acute kidney injury. Crit Care. 2007;11(2):R31. doi: 10.1186/cc5713. [PubMed: 17331245].

30. Schwartz GI, Gauthier B. A simple estimate of glomerular filtration rate in adolescent boys.JPediatr. 1985;106(3):522-6. doi: 10.1016/S00223476(85)80697-1. [PubMed: 3973793].

31. Bellomo R, Bagshaw S, Langenberg C, Ronco C. Pre-renal azotemia: a flawed paradigm in critically ill septic patients? Contrib Nephrol. 2007;156:1-9. doi: 10.1159/0000102008. [PubMed: 17464109].

32. Basu RK, Wong HR, Krawczeski CD, Wheeler DS, Manning PB, Chawla LS, et al. Combining functional and tubular damage biomarkers improves diagnostic precision for acute kidney injury after cardiac surgery. J Am Coll Cardiol. 2014;64(25):2753-62. doi: 10.1016/j.jacc.2014.09.066. [PubMed: 25541128]. 